\title{
Protecting Informative Messages over Burst Error Channels in Chain-based Wireless Sensor Networks
}

\author{
Zahra Taghikhaki $^{1}$, Nirvana Meratnia ${ }^{1}$ and Paul Havinga ${ }^{1}$ \\ ${ }^{I}$ Pervasive System Group, University of Twente, The Netherlands \\ \{Z.taghikhaki, N.meratnia,P.j.m.havinga\}@utwente.nl
}

Keywords: Wireless sensor networks, Reliability, Information-value, Adaptive error control, Information-aware, FEC.

\begin{abstract}
Regardless of the application, the way that data and information are disseminated is an important aspect in Wireless Sensor Networks (WSNs). The wireless data dissemination protocol should often guarantee a minimum reliability requirement. In this regard and to well-balance the energy and reliability, the more important packets should be protected by more powerful error control codes than the less important ones. This information-aware capability allows a system to deliver critical information with high reliability but potentially at a higher resource cost. In this paper, we first find and evaluate the factors that may influence the importance level of a packet and then design an error control approach by adaptively selecting codes for each individual links which experience long-term-fading and for each individual packet at run-time instead of applying network-wide settings prior to deployment. Moreover, we target the poor-explored chain-based topology that is of interest for many applications (e.g. monitoring bridge, tunnel, etc.). Simulation results validate the superiority of our approach compared with a number of Reed-Solomon-based error control approaches.
\end{abstract}

\section{INTRODUCTION}

Adhering to the packet-level or data constraints while designing a data disseminating protocol for WSNs may improve the system performance.

Most telecommunication systems use a fixed channel code to tolerate the expected worst-case error rate, which implies that they fail to operate at all if the error rate is worsened. The wireless channel is typically time varying and can exhibit high error rate over time. In order to improve the reliability of the data which is transmitted in WSNs, the error control approaches such as ARQ and FEC can be applied. Putting their advantages aside, existing error control techniques contribute to increase of the energy consumption due to the redundant data to be transmitted. Since energy is a scarce resource in WSNs, the type and the strength of the error control in use should be dependent on the type of the application. Generally speaking, event detection applications of WSNs need to execute more efficient and powerful error control techniques compared with periodic monitoring applications. However, the distinction between different packet type as being transmitted in these two classes of applications (periodic data and alarm) is neither general enough nor captures some important cases (e.g. the effect of channel condition or aggregation function) in WSNs. Therefore, even within a specific class of application, it would not be a proper to use a single error control code for all packets regardless of their different channel conditions or importance of information they carry. It is quite likely that even two packets both of which carry periodic monitoring data, not have the same amount of information and importance. For example, in a chain-based WSN data aggregation mechanisms are often used along the path with the aim of reducing the number of transmitted packets. Therefore, some packets may contain the aggregated readings of many nodes. These packets thus should be sent more reliably as they carry more informational value. It would be therefore a good idea to classify packets on the basis of their information-value based on which a proper error control scheme can be applied. By doing so, more important packets that have relatively high information-value are transmitted more reliably than packets carrying less important information. This is to well-balance the energy expenditure (caused by data and parity packets) and reliability. It is worth mentioning that by information-value we mean the amount of information a packet may have for the base station. Having dynamics of WSN into mind, adopting an efficient and accurate network-wide 
error control approach prior to network deployment is almost impossible. A very weak error control approach may not be able to correct many errors while a too strong code results in waste of time and energy resources. Dynamic error control schemes which are allocating the correctional power in an ondemand manner based on both the information-value and channel state are viable alternatives to static error control schemes, where the link conditions or packets' information-values are not taken into account. In this way and for the sake of efficiency, the information-value of a packet can be put into perspective with the amount of effort (in terms of energy expenditure) that is required to reliably transmit the given packet. Furthermore, since the wireless channel is inherently lossy and often manifests itself with bursts errors correlated in time, a reliable data dissemination should be capable of counteracting a large number of consecutive or burst errors. Since the application of run-time information-aware adaptive error control mechanisms for WSNs operating under timely and spatially variable channel conditions has generally been less-studied, in this paper we give emphasize to this type of application. In this paper, first the factors that may influence the information-value of a packet will be investigated. Then we incorporate all these obtained factors in order to estimate the informationvalue of the packets. Finally, we exploit the information-value as a means to properly adjust the parameters of the adaptive error control code in use. In this regards, we propose RAFEC*, which is a Run-time Adaptive FEC-based data dissemination protocol to enhance reliability, based on the amount of information the packets carry over a long-term error-bursty channel in a chain-based WSN. This adaptation gives the possibility to vary the code strength and complexity on-demand and on the fly.

One should not that the targeted topology in RAFEC* is chain topology. Importantly, there is not much work on reliable data dissemination in chainbased wireless sensor networks and thus there are some areas to which special attention should be paid. Even though many reliable data disseminating protocols have been designed for wireless sensor networks (Al-Karaki and Kamal 2004), most of them are usually designed for a general topology such as mesh which work well in a multidimensional deployment. For applications with linear topology, in which nodes are usually lined up in one-dimensional formation, however, a mesh topology may not be appropriate or simply not feasible due to the physical structure or measuring point distribution, among others. Moreover, it is a good idea to take the advantage of a linear topology over a predetermined linear infrastructure (e.g. bridge, tunnel, etc.), which may be quite different than a randomly deployed network.

\subsection{The need for packet-level FEC}

Basically, FEC applied at the bit-level and bytelevel is appropriate for short-term errors and additive white Gaussian noise when rapid fluctuation is experienced over a short period of time. This is because in this situation, only some bits or bytes of a packet are influenced. FEC applied at bit- or bytelevel is less efficient in recovery from burst bit errors caused by long-term fading and expanded over several packets. In this regards, it is unable to recover a completely lost or delayed packet. Therefore, in these cases either ARQ or a packetlevel FEC should be employed. ARQ-based approaches are effective only for a shorter time-scale or short-term burst errors. In this respect, even though ARQ could tolerate long-term fading to some extent, but more persistent fluctuations make this approach as inefficient as bit- and byte-level FEC. To overcome the unreliability caused by more persistent fluctuations or long-term burst errors, application-level or packet-level FEC may be used.

The rest of this paper is organized as follows. First we explain the assumption and model we used in Section 2, which is followed by the related work in Section 3. Then in Section 4, we describe the problem statement and our contribution. We elaborate on our proposed RAFEC* protocol in Section 5. Then in section 6 we present the simulation setup and performance evaluation results. Finally in Section 7 we draw the conclusion.

\section{ASSUMPTIONS AND MODELS USED}

We make the following assumptions regarding the WSN:

- The WSN consists of $\mathrm{N}$ sensor nodes uniformly and randomly deployed in a chain topology.

- The channel is considered to vary slowly with respect to the data transmission rate, and thereby the channels state transitions occur infrequently.

- A systematic code is preferred, as it less suffers from delays imposed by the block code mechanisms.

- Uncertainty parameters of the nodes and links are fixed over transmitting a single code-word.

- The transmission errors are assumed to be local and spatially and temporally variable, which in 
turn should be tackled on a per-link and not network-wide basis.

\subsection{Channel model}

In wireless networks, the cause of packet loss can become more complex and dynamic so that the frequency of the error bursts varies over time. We use a Quasi-Stationary Gilbert-Elliot (QSGE) model, as shown in Figure 1, in order to model channel states. Each state $S_{\mathrm{v}}$ which corresponds to a specific packet error rate $P E R_{v}$ follows a Gilbert-Elliot model with some probabilities ( $p$ and $q$ ) associated to it. The B (Bad) and G (Good) states are also a series of Bernoulli trials.

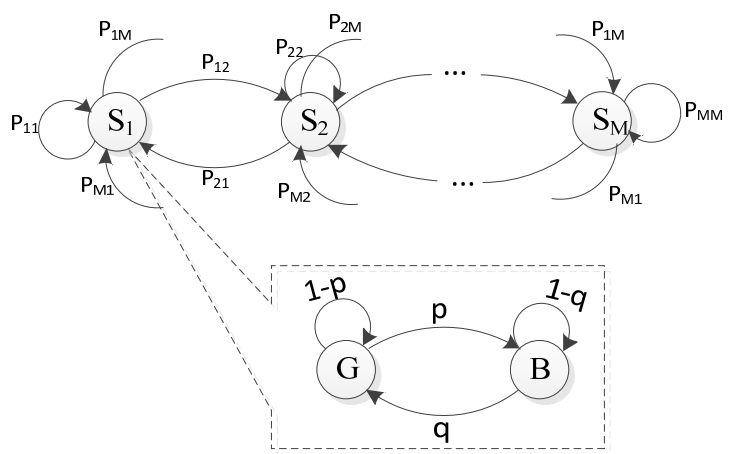

Figure 1. Quasi-stationary Gilbert-Elliot model

Each state $\mathrm{S}_{\mathrm{i}}$ represents the expected PER $r_{p}^{i}$ so that $r_{p}^{1}<r_{p}^{2}<\cdots<r_{p}^{M}$, and the conditional one step probabilities of going from channel state $S_{i}$ to channel state $\mathrm{S}_{\mathrm{j}}$ is given by $P_{i j}$. The channel could be described in form of a transition matrix with entries as cross over probability over all combination of states. The corresponding state transition matrix ( $\left.\Gamma=\left\{P_{i j}\right\}\right)$ of the 0 , that governs the process of how the channel introduces different error rates, is expressed as:

$$
\Gamma=\left[\begin{array}{ccccc}
P_{11} & P_{12} & \ldots & P_{1(M-1)} & P_{1 M} \\
P_{21} & P_{22} & \ldots & P_{2(M-1)} & P_{2 M} \\
P_{31} & P_{32} & \ldots & P_{3(M-1)} & P_{3 M} \\
\vdots & \vdots & \ldots & \vdots & \vdots \\
P_{M 1} & P_{M 2} & \ldots & P_{M(M-1)} & P_{M M}
\end{array}\right]
$$

In order to simulate slowly varying channel the following relationship among transition probabilities should be presented:

$$
\begin{array}{rlrl}
P_{i, j} \gg P_{i, j+1} \gg P_{i, j+2} & \gg \cdots \gg P_{i, M} & i \leq j, \forall i, j \\
& \in[1, M] & \\
P_{i, j} \gg P_{i, j-1} \gg P_{i, j-2} & \gg \cdots \gg P_{i, 1} & i>j, \forall i, j \\
& \in[1, \mathrm{M}] &
\end{array}
$$

\section{RELATED WORK}

Although numerous research have been published related to error control in wireless networks, especially in cellular networks, most of these are not directly applicable to WSNs. The limited energy, low complexity of the sensor node hardware, and harsh/dynamic environment of the deployment area necessitates an energy-efficient and more dynamic or adaptive error control strategy to be used.

The adaptive reliable data dissemination protocols typically fall in two main categories:

(i) Link-aware: This category of protocol including (Comroe and Costello Jr 1984; Ahn, Hong et al. 2005; Charfi, Wakamiya et al. 2007; Liankuan, Deqin et al. 2010; Eriksson, Bjornemo et al. 2011; Hurni and Braun 2011; Yu, Barac et al. 2012) (Yan-ming, Yong-jun et al. 2009; Taghikhaki, Meratnia et al. 2012; Taghikhaki, Meratnia et al. 2013) propose error control schemes whose correction capability vary according to the links quality.

(ii) Information-aware: The basic idea of this category of protocols including (Deb, Bhatnagar et al. 2003) (Deb, Bhatnagar et al. 2003) (Bhatnagar, Deb et al. 2001; Karl, Löbbers et al. 2003) (Kopke, Karl et al. 2005) (Kleinschmidt, Borelli et al. 2009; Kleinschmidt and da Cunha Borelli 2009) is that not all 'to be transferred' packets require $100 \%$ reliable delivery. Instead, the reliability is application-specific and reliability requirements depend on the different importance levels of packets or environmental conditions. The advantage offered by this category of protocols is that limited resources, such as bandwidth and energy, will only be spent on important information with high-reliability requirements. These protocols basically rely on information-awareness and consider diverse priorities among different packets. The novelty of these approaches is that they consider the need for information-awareness and adaptability to the link quality along with allocation of network resources based on the criticality of data. Each priority level is usually mapped to a desired reliability for data delivery.

Most of these approaches assume a simple independent loss channel, which is modeled by Bernoulli distribution and therefore they usually fail to be applied in error-bursty channels.

Basically, all packet transmissions in these approaches have the same probability to fail and each transmission error is independent from the others. However, wireless channel is inherently 
lossy and often manifests itself in the form of burst errors correlated in time. Therefore, a reliable data dissemination should be capable of counteracting long-term fading possibly extending over several packets because of high concentrations of errors. To cope with this issue, a packet-level adaptive forward error correction may be a good alternative.

Some approaches rely on the multiple path transmission, which are highly dependent on the network topology. In a chain-based topology where the communication of a sensor node is often restricted only to its immediate neighboring nodes (i.e. successor and predecessor node), we cannot well-benefit from the availability of multiple paths to salvage data packets from node/link failures. In case of using duplicate-sensitive aggregation functions such as SUM or AVERAGE, these multipath approaches should employ some more resource demanding methods to filter out the redundant data. Moreover, these approaches require to some extent ensure that only one of the upstream neighbors forward the packet copies through multi-paths, otherwise they will introduce large amount of traffic, which leads to waste of resources in case all upstream neighbors send multiple copies. To strictly enforce that only one of the upstream nodes transmit the packet copies, these approaches may either incur extra overhead in the form of some control packets or use some probabilistic methods to lower down the probability of transmitting a packet by the upstream nodes (Deb, Bhatnagar et al. 2003).

Majority of the information-aware protocols do not evaluate the information-value of the packets and assume that sensor nodes have a priori knowledge to determine the importance level of the packets. Using these approaches, when a source node initiates a packet, it should set the importance level (or information-value) of the packet. However, asking sensor nodes to determine the importance level of the sensory data introduces new challenges which may require complex algorithms to perform pattern matching or execute artificial intelligence techniques. Moreover, in these approaches the importance level of each packet is set once on the source node and does not change along the path. Therefore, if an important sensory data is modified along the path in such a way that it cannot anymore reflect the phenomena state, transmitting it leads to wasting sensor/network resources. To cope with this issue, the importance level of the packets should vary along the path by considering the factors which may influence the packet importance level.

Some approaches specially those which consider the aggregation degree to determine the importance level of the packets, poorly perform in case of being applied in uniformly distributed deployments. Nonuniform and unevenly distribution of sensor nodes results in some areas to be monitored by many sensors while other areas will be monitored only by a few nodes. Therefore, considering just the aggregation degree of the nodes may not well-reflect the importance level of the data. In this regard, the information-value of the packets should be determined in such a way that could also be applied for non-uniformly distributed deployments.

The above discussion highlights the need for an adaptive reliable chain-based disseminating protocol based on both packet information-value and link quality. To this end and to address most of above shortcomings, an adaptive energy-efficient reliable disseminating protocol is needed which (i) can be applied to non-uniform deployments with linear topology (ii) tackles the long-term error bursts, (iii) incorporates various factors that may influence the information quality of the packets, and (iv) considers packet delivery ratio as the link quality metric, rather than considering immediate channel quality indicators such as RSSI and SNR which are not appropriate for long-term error burst.

\section{PROBLEM STATEMENT AND OUR CONTRIBUTION}

Given an already deployed linear WSN, the problem at hand is to design an adaptive, reliable, energyefficient, and Information-Link-aware data dissemination protocol. We summarize our contribution related to this paper as: (i) Investigating and quantifying different factors which may influence the information-value of packets and incorporating the above identified factors in evaluation of informational content and importance of packets. (ii) proposing RAFEC*, i.e., an adaptive, energy-efficient, reliable, information-link-aware data dissemination approach, which is able to (a) cope with periodic long-term loss process in a linear chain-based WSNs and (b) switches among error control codes with different powers to vary the code strength and complexity in on demand.

\section{RAFEC*}

In this section we elaborate on RAFEC*, which is a Run-time Adaptive FEC-based data dissemination protocol that improves reliability of packet delivery based on the amount of information they carry over a bursty channel in a chain-based WSN. To this end, (i) the mechanism for associating the error control codes to the states of QSGE model is described (ii) packet information and link quality 
are estimated and (iii) the strategy using which an appropriate error control code is assigned to a specific packet is explained.

Basically, the activities performed by every sensor node $i$ can be organized into sequences each of which may correspond to processing one codeword $c w_{j}^{i}$ as shown in Figure 2.

\subsection{Assigning error control codes to the channel states}

As we stated before, in RAFEC* the channel is modeled as a M-states QSGE model with a packet error rate $P E R_{s}$ assigned to each state $\mathrm{S}_{\mathrm{s}}$. Therefore, at any moment of time the state of the channel should fit one of the states specified by the channel model.

Having the packet error rate $\mathrm{PER}_{\mathrm{s}}$ of each state $\mathrm{S}_{\mathrm{s}}$ of the M-state QSGE model, an error control code which can effectively counteract the available errors may be designed. To this end, the error control codes in RAFEC* are selected from a single family of FEC block codes such as $F_{O} B_{R S}(n)$ which represented a family block code for a Reed-Solomon code:

$$
F_{o B} B_{R S}(n)=\{R S(n, k) \mid k=n-2 \times t, k>0\}
$$

where $k$ represents number of original data and $t$ represents correction capability of the ReedSolomon code $R S(n, k)$. Each member of family block $F_{O} B_{R S}(n)$ can correct up to a specific number of error $t$. RAFEC* uses $F_{o} B_{R s}(n)$ for the M-state QSGE model. Therefore, each state $\mathrm{S}_{\mathrm{s}}$ of the M-state channel, which exhibits a specific error rate $P E R_{S}$, can adopt one member of $F o B_{R S}(n)$ based on the below Equation provided that $\left|F_{O} B_{R S}(n)\right| \geq M$ :

$$
\begin{gathered}
\mathrm{ECC}_{s}=\mathrm{RS}\left(\mathrm{n}, k_{s} \geq K_{s}\right) \\
\mathrm{RS}\left(\mathrm{n}, k_{s} \geq K_{s}\right) \in F o B_{R s}(n) \\
K_{s}=n \times\left(1-P E R_{s}\right)
\end{gathered}
$$

To this end, the most efficient error control code denoted by $\mathrm{ECC}_{\mathrm{s}}$ which exhibits the "just enough" correctional power for the channel state $\mathrm{S}_{\mathrm{s}}$ is $R S$ ( $n$, $\left.K_{s}\right)$. In this way, each channel state $\mathrm{S}_{\mathrm{s}}$ can be described using two parameters $P E R_{s}$ and $K_{s}$ as $\mathrm{S}_{\mathrm{s}}\left(P E R_{s}, K_{s}\right)$. In short, a particular coding strategy $E C_{s}$ is associated with each channel state $S_{s}$. The criteria by which this coding strategy is selected is addressed in above Equation.

\subsection{Assessing packet information and link quality}

Since the choice of error control code for each packet in RAFEC* is based on the quality of service parameters, the information-value and packet importance as well as properties of error traces which are captured from transmission history, the following tasks need to be performed by the sensor nodes:

- Estimation of packet's information value

- Estimation of link quality

\subsubsection{Estimation of information-value}

The information-value could be influenced by several factors which may have different priorities in different applications. In what follows we express these factors which we then take into consideration to estimate information-value of a packet.

- Node functionality: Faulty sensor nodes could influence network operation and pose a challenging constraint in the design of a protocol for WSNs. Most of reliable data dissemination protocols usually concentrate on the link quality and less effort has been put into the node's functionality. Having a reliable dissemination protocol by itself is not useful if relay nodes through which data is disseminated are faulty and malfunctioning. Therefore, it is important that all sensor units relevant to the accomplishing task operate well-enough in order to ensure high reliability. In this regard, the quality of sensing and computing unit of relay nodes should be considered when estimating packet informationvalue. To estimate the quality of sensor units, we use a trust-based approach as introduced in (Taghikhaki, Meratnia et al. 2013). 


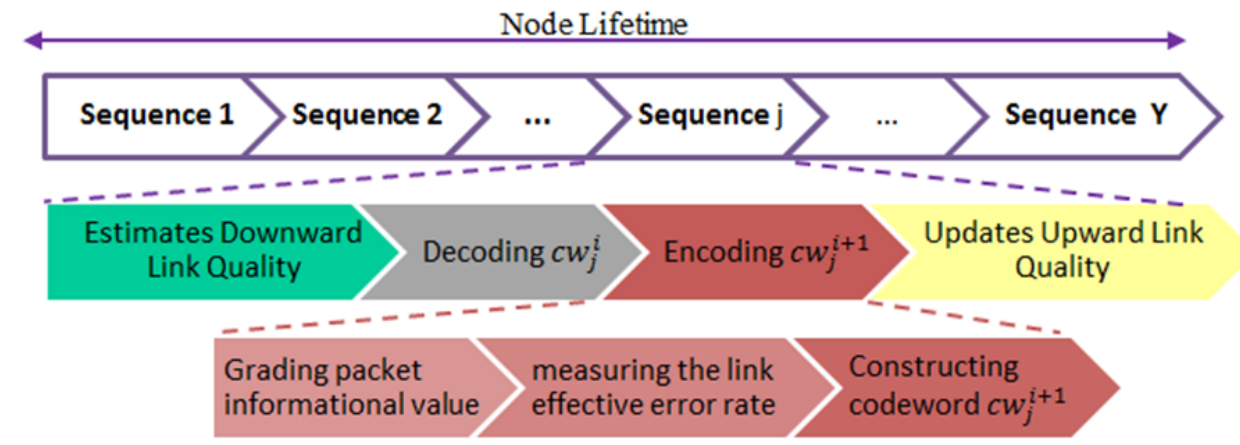

Figure 2. Activities performed by relay node $i$

- Node contribution degree: The relative position of each node in the network may also impact the information-value of a packet being disseminated through the given node. Generally speaking, the higher contribution degree $\psi$ of a node, the higher information-value. As can be seen from Figure 3, contribution degree of node $S_{7}$ is higher than $S_{12}$ as it monitors three critical points $\left(\psi\left(S_{7}\right)=3\right)$ while $\mathrm{S}_{12}$ only monitors one critical point $\left(\psi\left(S_{12}\right)=1\right)$. Node contribution degree is determined by the base station which informs each node about its $\psi$.

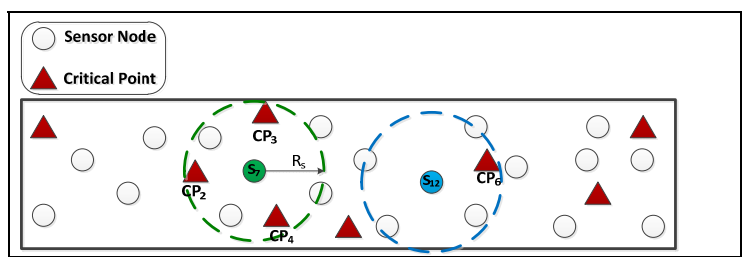

Figure 3. Illustrative example of node contribution degree

- Node spatial density: If sensor nodes are not evenly distributed, it is likely that some sensor nodes simultaneously and so redundantly observe a critical point while some nodes only and lonely observe a critical point. In this case, relying only on the coverage degree does not well reflect the amount of information being sent by the sensor nodes. As can be seen from Figure 4 although $\psi\left(S_{2}\right)<\psi\left(S_{15}\right), \mathrm{S}_{2}$ is the only node which can observe critical point $\mathrm{CP}_{1}$ while critical point $\mathrm{CP}_{6}$ is being monitored by other three nodes in addition to node $S_{15}$. Therefore, a sensory data coming from a region that is already covered (either fully or partially) by other nodes has less informative content. On the other hand, if a sensor node is located in such a place where it covers one or some critical points which are not been observed by any otherwise node, its sensed data more likely carries quite significant information. Node spatial density can easily be determined by the base station in the initialization phase and then the base station informs each node about it.

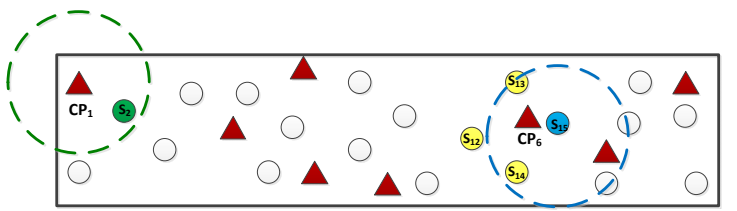

Figure 4. Illustrative example of node spatial density

- Strategic Area: The value of data collected from different critical regions may not necessarily be equal. A given application (either always or sometime) may be more interested in data of some specific cells/regions. Therefore, the informationvalue of packets carrying this data is higher. As it can be seen from Figure 5 although node $\mathrm{S}_{6}$ monitors two critical points (i.e., $\mathrm{CP}_{2}$ and $\mathrm{CP}_{4}$ both having importance of 1 ) and node $S_{13}$ monitors one critical point $\mathrm{CP}_{6}$ having importance of 4 , information-value of data coming from node $\mathrm{S}_{13}$ is higher as it covers a more strategic area. In the initialization phase, the base station informs each node about the strategic level (or criticalness) of an area in where the given node is located.

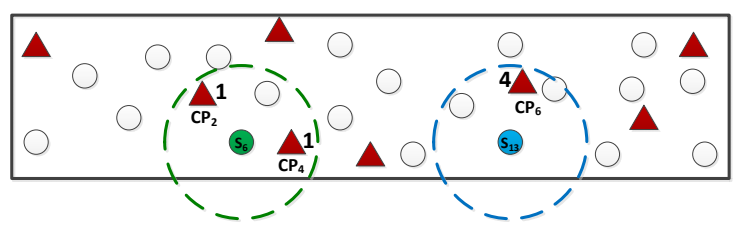

Figure 5. Illustrative example of different strategic area

- Traveled Distance Ratio: If a packet is lost at the first hops, (i) lesser energy has been consumed for its relay and (ii) lesser information (in case of doing aggregation along the path) are lost, compared to when it is lost at further hops. Therefore, it makes sense to use stronger error control codes for packets being relayed for longer distance. This parameter can be determined by 
increasing a counter (a packet's field) whose value is zero in the source node.

After identifying the aforementioned factors that may impact the quality of data packets, we here explain how to estimate the information-value and importance of packets per hop.

In Equation (3) we combine all aforementioned factors except Traveled Distance Ratio. The reason to leave Traveled Distance Ratio out of this equation is that all other factors are node-dependent while Traveled Distance Ratio is both packet-dependent and node-dependent.

$$
\xi(i)=\sum_{k \in \operatorname{SCov}_{i}} \frac{\sigma_{k} \times \gamma_{i, \mathrm{k}}^{\text {total }}}{\left|\operatorname{CCov}_{k}\right|}
$$

where CCovk represents the set of nodes that cover the common/critical point $k$, SCovi states set of common/critical points which have already been covered by sensor node $i, \sigma_{\mathrm{k}}$ denotes how critical and strategic the data of common/critical point $k$ is, and $\gamma_{\mathrm{i}, \mathrm{k}}^{\text {total }}$ signifies node $i$ functionality which is obtained from (Taghikhaki, Meratnia et al. 2013). for common/critical point $k$.

To also take Travelled Distance Ratio into account, we utilize Equation (4), where SIDp represents $I D$ of the source node which initiates the data packet $p$. The numerator evaluates the travelled distance while the denominator represents the distance between the base station (BS) and the source node.

$$
\vartheta(i, p)=\frac{\left|i-S I D_{p}\right|}{\left|B S-S I D_{p}\right|}
$$

Exploiting Equation (3) and (4) further, information-value denoted by $\chi(p, i)$ of packet $p$ being sent by sensor node i can be calculated using Equation (5).

$$
\chi(p, i)= \begin{cases}\frac{\chi(p, i-1)+\varpi_{1} \times \vartheta(i, p)+\varpi_{2} \times \xi(i)}{\hat{\chi}} & i \neq S I D_{p} \\ \frac{\xi(i)}{\hat{\chi}} & i=S I D_{p}\end{cases}
$$

where $\grave{\chi}$ represents maximum information-value that a data packet may have.

Weights $\left(\varpi_{1}, \varpi_{2}\right)$ used in Equation (5) can be adjusted according to the application specific knowledge. For instance, if the application does not perform aggregation on the intermediate nodes and thus the relay nodes carry only the raw data, we may set $\varpi_{1}=1$ and $\varpi_{2}=0$. For the sake of simplicity and without loss of generality, we can map packet's information-value denoted by $\chi$ into $\mathrm{d}_{\mathrm{v}}$ discrete values. Doing so, we will have $d_{v}$ different packet types each of which contains a specific amount of information and thus their required reliabilities are different. Therefore, $d_{v}$ also shows the number of required error control codes each of which is assigned to a specific information-value. In this thesis by using Equation (6), we map packet's information-value into discrete values 1, 2 and 3. By doing so, three different packet types will be defined in terms of information-value they may have. However, depending on the available error control codes which are implemented in the sensor nodes we can have different values for $\mathrm{d}_{\mathrm{v}}$.

$$
\gamma=\left\{\begin{array}{rr}
1 & 0 \leq \chi<0.3 \\
2 & 0.3 \leq \chi<0.6 \\
3 & 0.6 \leq \chi
\end{array}\right.
$$

\subsubsection{Estimation of link quality}

To estimate the link quality, RAFEC* employs a passive link monitoring strategy, which exploits existing traffic without incurring additional communication overhead.

The link quality estimation process in RAFEC* is performed first over a sequence of $\omega$ packets (say packet-level estimation) and then over a sequence of $\widehat{\omega}$ code-words (say code-word-level estimation).

Having statistics about a given link qualities over the last sliding window, we calculate the average error rate $\overline{r \varphi}$.

As will be stated later, dependent on amount of information a packet carries, we change $\widehat{\omega}$ in order to capture the effective-error-rate on the links.

\subsection{Adaptive packet-link-local error control}

Having both information-value of packets and packet error rates captured from transmissions history, strength and complexity of the error control codes can be adapted on demand. Having higher information-value or poorer link quality requires utilization of a more powerful error control code. On the contrary, having lower information-value or higher quality link requires a weaker code.

It is noteworthy that we consider a multi-hop FEC protection mechanism, in which intermediate nodes need to perform encoding and decoding functions individually and locally at each hop. This way of locally protection helps our approach being easily applied to large-scale networks.

In Section 5.1, we explained how Reed-Solomon code is assigned to each channel state of QSGE model based on the packet error rate $P E R_{s}$ of each state $\mathrm{S}_{\mathrm{s}}$. Basically, effective-error-rate $\varphi(u)$ of a link at any moment of time $u$ should correspond to one of the $P E R_{S}$ specified by the QSGE model.

Then according to Equation (7), the error control code $\mathrm{ECC}_{\mathrm{s}}$, which is associated to the state $\mathrm{S}_{\mathrm{s}}$ could 
be decided as the code $\operatorname{EEcc}(\varphi)$ that should be utilized for the error rate $\varphi$.

$$
\operatorname{EEcc}(\varphi)=E C C_{s}
$$

Our strategy to estimate the effective error rate $\varphi$ can be summarized as:

- First, we calculate the average error rate for three different values (i.e. 1,2,3) assigned to $\widehat{\omega}$. In this regard, dependent on the $\widehat{\omega}$ value, three different average error rates $\overline{r \varphi}(\widehat{\omega})$ may obtain. According to Equation (8) we put each of these three values to a variable $\varepsilon_{\widehat{\omega}}$.

$$
\begin{array}{ll}
\varepsilon_{1}=\overline{r \varphi}(\widehat{\omega}) & \widehat{\omega}=1 \\
\varepsilon_{2}=\overline{r \varphi}(\widehat{\omega}) & \widehat{\omega}=2 \\
\varepsilon_{3}=-\overline{r \varphi}(\widehat{\omega}) & \widehat{\omega}=3
\end{array}
$$

- Second, having Information-value of the packet we estimate the effective error rate $\varphi$ for each packet as:

- If information-value of the packet is $\gamma=1$ then the effective error rate $\varphi$ will be $\varepsilon_{1}$.

- If the packet has higher information-value $(\gamma=2)$ then $\varphi$ will be $\operatorname{Max}\left(\varepsilon_{1}, \varepsilon_{2}\right)$.

- If the packet has the highest informationvalue $(\gamma=3)$, the effective packet error rate $\varphi$ will be $\operatorname{Max}\left(\varepsilon_{1}, \varepsilon_{2}, \varepsilon_{3}\right)$.

According to above, the effective packet error rate $\varphi$ based on which the error control code is selected, varies according to the information-value $\gamma$. In this regard, a packet with high/low information-value should be equipped with a strong/weak error control code EECC presented in Equation (7).

\section{PERFORMANCE EVALUATION}

\subsection{Performance metrics}

We consider the following metrics to evaluate the performance of our approaches under different circumstances.

- Information-aware reliability ratio (IRR): This metric evaluates reliability ratio by taking information-value of the packets into account using Equation (9):

$$
\operatorname{IRR}(\gamma)=\frac{\sum_{i=1}^{\bar{N}_{s}-1} N_{\gamma}^{R e f}(i+1)+\sum_{i=1}^{\bar{N}_{s}-1} N_{\gamma}^{R a R}(i+1)}{\sum_{i=1}^{\bar{N}_{s}-1} N_{\gamma}^{T D}(i)}
$$

where $\bar{N}_{S}$ represents the number of sensor nodes. Moreover, $N_{\gamma}^{T D}(i), N_{\gamma}^{R e f}(i)$ and $N_{\gamma}^{R a R}(i)$ are the number of data packets with information-value $\gamma$ transmitted by node $i$, received by node $i$ error-freely and correctly being recovered by node $i$, respectively. According to Equation (9), we will have three different IRRs each of which representing the achieved reliability ratio for a specific information-value $\gamma$.

- Code rate: This metric represents the proportion of the useful (non-parity) packets in a code-word. By the means of this metric, we express the code's efficiency and the redundancy introduced by the code.

- Information-aware System Efficiency : It is generally accepted that additional parity packets (or lowering the code rate) can be tolerated as long as loss-resiliency at the receiver side is increased. Therefore, the system efficiency metric is introduced to express the tradeoff between the energy expenditure and reliability. To this end we make a relation between information-value arriving at the destination with the amount of redundancy (parity packets) and define Equation (10) as:

$I S E=\sum_{\gamma=1}^{3} v(\gamma) \times \frac{\sum_{i=1}^{\bar{N}_{s}-1} N_{\gamma}^{R e f}(i+1)+\sum_{i=1}^{\bar{N}_{s}-1} N_{\gamma}^{R a R}(i+1)}{\sum_{i=1}^{\bar{N}_{s}-1} N_{\gamma}^{T D}(i)+\sum_{i=1}^{\bar{N}_{s}-1} N_{\gamma}^{T R}(i)}$

where $N^{T R}$ represents the number of redundant (parity) packets sent by node $i$. and $v(\gamma)$ represents the amount of gain that an application earns by receiving a packet with an informationvalue $\gamma$. We assume that the gain of a packet with $\gamma=3$ is twice of that for $\gamma=2$ and four times of that for $\gamma=1$. Therefore, $v(\gamma=3)=2 \times$ $v(\gamma=2)=4 \times v(\gamma=1)$. By doing so, receiving a packet with information-value $\gamma=2$ worth twice as much as receiving a packet with information-value $\gamma=1$.

\subsection{Simulation setup and scenario}

We consider a chain consists of 20 nodes which are linearly deployed in an area of $400 \mathrm{~m} \times 25 \mathrm{~m}$ and in all simulations, the source or initiative node is the leftmost node. The sensing range of nodes is to $35 \mathrm{~m}$. Unless otherwise states, the simulation parameters are as described here. The deployment area is divided into some regions $(l=25 \mathrm{~m})$ half of which are labeled as critical and the rest are labeled as uncritical. It is worth mentioning that since RAFEC* is a link-local error control approach, the number of sensor nodes does not much influence the performance of the application. We then send 5000 
packets from one source node to the base station with frequency of $1 \mathrm{pkt} / \mathrm{s}$. The strategic level (or criticalness) of the critical regions is selected from the interval (Bhatnagar, Deb et al. 2001) while the strategic-level of the uncritical regions are 1 . At any moment in time, $70 \%$ of all nodes and links work almost properly with failure rate of 0.09 . The failure rate of other $30 \%$ of the nodes is set to 0.85 . The failure rate of other $30 \%$ of the links vary according to a five-state QSGE model which will be state later. The selection of failing nodes/links occur randomly after every 1000 time unit in order to simulate temporal correlation among failures of those $30 \%$ nodes/links.

Five-states QSGE erasure channel (as explained in Section 2) is used. In order to simulate a slowly varying channel, the following specifications are used:

$$
\Gamma=\left[\begin{array}{ccccc}
0.995 & 0.0035 & 0.0015 & 0 & 0 \\
0.0033 & 0.992 & 0.0033 & 0.0014 & 0 \\
0.0015 & 0.0025 & 0.992 & 0.0025 & 0.0015 \\
0 & 0.0014 & 0.0033 & 0.992 & 0.0033 \\
0 & 0 & 0.003 & 0.007 & 0.99
\end{array}\right]
$$

The probability of staying in one state, i.e. $\mathrm{P}_{\mathrm{i}, \mathrm{i}}$, is extracted from (Rice and Wicker 1994) and the remainder, i.e. 1- $P_{i, j}$, is evenly allocated to transitions from node $i$ to all other nodes $j(i \neq j)$ so that $\sum_{j=1}^{9} P_{i, j}=1-P_{i, i} \quad \forall i \in[1,9],(i \neq j)$.

Each state $\mathrm{S}_{\mathrm{s}}$ of the five-state QSGE model corresponds to one $\mathrm{PER}_{\mathrm{s}}$ as: $\mathrm{PER}_{1}=0.1, \mathrm{PER}_{2}=0.3$, $\mathrm{PER}_{3}=0.4, \mathrm{PER}_{4}=0.5, \mathrm{PER}_{5}=0.7$.

We model sending packets in each state of QSGE model first according to a Gilbert-Elliot model and then as a series of Bernoulli trials. The Gilbert-Elliott channel model is defined by $p$ and $q$ which change according to the $N$ and $N-K$ parameters of the codes assigned to $S_{s}$ (Table 1) These two parameters are obtained as:

$$
\begin{aligned}
& \mathrm{p}^{1}=0.07, \mathrm{p}^{2}=0.09, \mathrm{p}^{3}=0.11, \mathrm{p}^{4}=0.14, \mathrm{p}^{5}=0.2 \\
& \mathrm{q}^{1}=0.5, \mathrm{q}^{2}=0.25, \mathrm{q}^{3}=0.166, \mathrm{q}^{4}=0.125, \mathrm{q}^{5}=0.1
\end{aligned}
$$

In our approach, a length-15 Reed-Solomon (RS) code (i.e. $\mathrm{N}=15$ ) is chosen over a five states channel for packets with three different information-values. The error control code $\mathrm{ECC}_{\mathrm{s}}$ which is assigned to each state $S_{s}$ is presented in Table 1 . The error codes contained in this table are increasing in their correctional power from the left to the right, and similarly with respect to computational and parity overhead.The information-value weights are set to $\varpi_{1}=1, \varpi_{2}=1$ and $\omega 1=\omega 2=\omega 3=1$.Moreover, the channel estimation windows size is $|\omega|=$ 15 while the sliding window size is $|\widehat{\omega}|=5$.

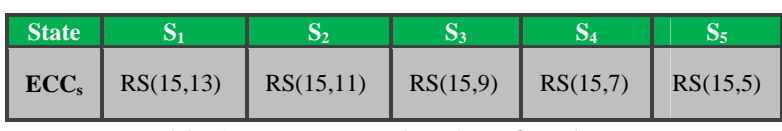

Table 1. Error control codes of each state

\subsection{Performance evaluation}

Figure 6. represents the IRR and each graph in this figure belongs to one specific packet error rate (PER) under which a packet that may carry different amount of information is transmitted. One can see that IRR of RAFEC* heavily depends on the information-value of the packet. The more informative packet (higher $\gamma$ ), the less likely the packet will be lost and so the higher contribution in the overall RR. In Figure 6 the relationship among the reliability ratio of different information-values in RAFEC* is:

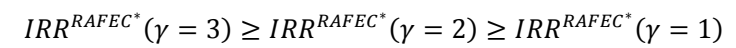

Following this intuition, the IRR of the most informative packets in RAFEC* are always maximum and greater than $90 \%$. Moreover, since packets with $\gamma=1$ are less important for the application, the RAFEC* does not use robust error control for them and thereby the IRR for them is relatively low. According to Figure 6, no fixed relationship among reliability ratio of different information-values for other approaches can be inferred and they just exhibit a very random behavior.

The average gained code rate for the received packets which carry different informative content is illustrated in Figure 7. The code rate of RAFEC* is inversely proportional to the packet error rate as RAFEC* needs to dynamically adjust the amount of parity packets to be able to overcome the incurred errors. Generally, the high error rate necessitates the use of more parity packets, which in turn results in a lower code rate. Since other approaches are all static, changing the error rate does not have any effect on the code rate. The code rates shown in Figure 7 are averaged over three informationvalues. To have a better insight about the obtained code rate per different information-value, Figure 8 is presented. The higher packet error rate necessitates to equip data-words with a more powerful code, which results in more parity packets and thereby lower code rate. Obviously, packet with $\gamma=3$ produces low code rate which explains its superior performance in terms of reliability. 

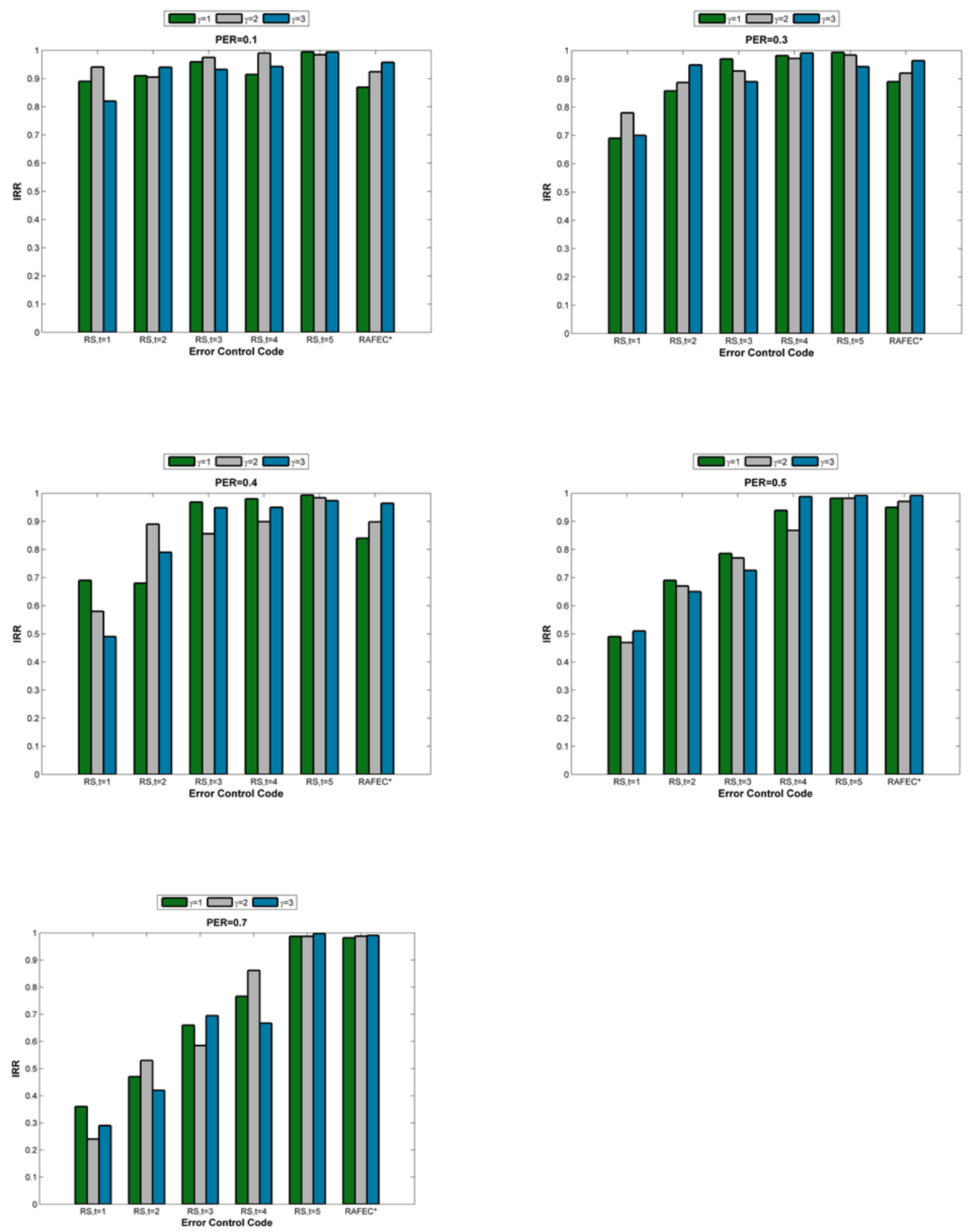

Figure 6. Information-aware reliability ratio for different packet error rate for RAFEC* and RSs 


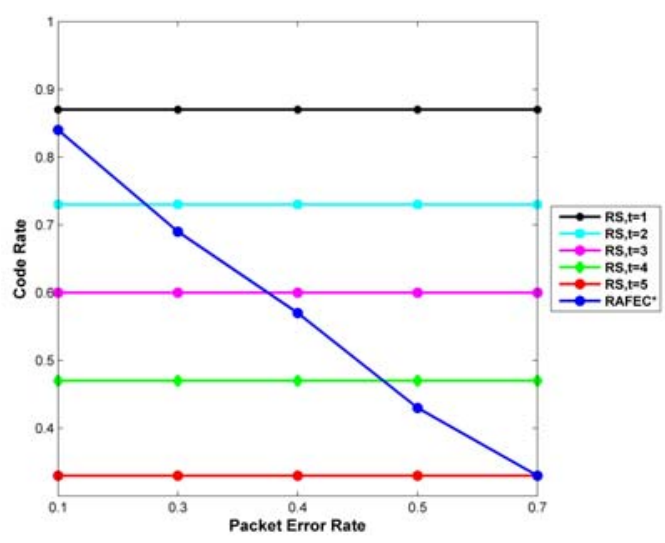

Figure 7. Code rate comparison of RAFEC* and RSs

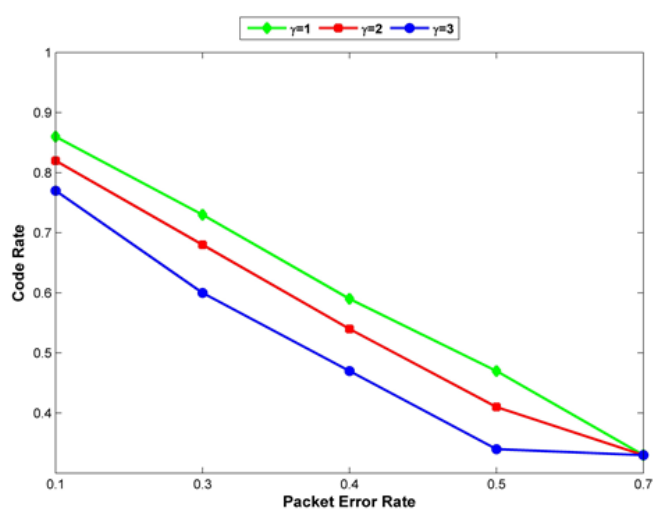

Figure 8. Code rate comparison of RAFEC*

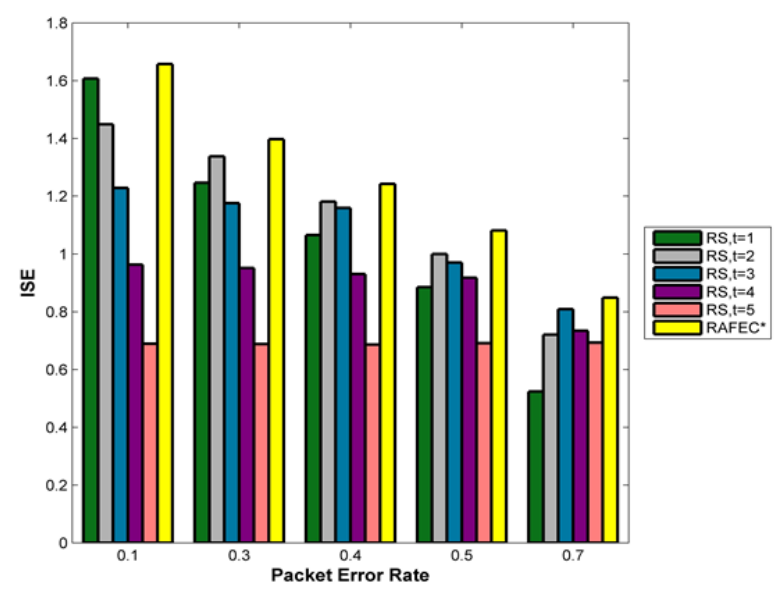

Figure 9. Information-aware system efficiency comparison of RAFEC* and RSs
Figure 9 illustrates Information-aware System Efficiency of different codes, form which superiority of RAFEC* over all other codes can be seen.

\section{CONCLUSION}

The purpose of WSNs is sensing and disseminating information. Therefore, the loss of important information at the perceived benefit of saving energy, may inhibit the ability of a WSN to fulfil its primary purpose.

In this paper, we propose RAFEC* a packetlevel reliable data dissemination protocol to support information-awareness in a chain-based WSN. Different from most of the proposed reliable approaches that are proposed to work for the topologies other than chain and so cannot efficiently work for the chain topology, RAFEC* is customized for this poor-explored topology. Using RAFEC*, information can be delivered at desired levels of reliability at proportional cost, in spite of the presence of long-term fading in the channel. RAFEC*, basically exploits the concept of dynamic packet state and dynamic link state to control the correction capability of the error control codes exploiting only local knowledge of channel and packets at each hop. Moreover, the history-based evaluating link quality which RAFEC* utilizes, provides a means to cope with longer-term interferences, since the mechanism does not immediately switch to a less/more powerful code after one successful/failed transmission. Basically, RAFEC* waits until a couple of transmission have succeeded or failed and then change the error control in-use.

In the simulation, we illustrate the superiority of RAFEC* in terms of several metrics.

\section{ACKNOWLEDGEMENT}

This work is supported by IST FP7 STREP GENESI: Green sEnsor NEtworks for Structural monItoring project.

\section{REFERENCE}

Ahn, J.-S., S.-W. Hong, et al. (2005). "An adaptive FEC code control algorithm for mobile wireless sensor networks." Journal of Communications and Networks 7(4): 489498. 
Al-Karaki, J. N. and A. E. Kamal (2004). "Routing techniques in wireless sensor networks: a survey." Wireless communications, IEEE 11(6): 6-28.

Bhatnagar, S., B. Deb, et al. (2001). Service differentiation in sensor networks. International Conference on Wireless Personal Multimedia Communications.

Charfi, Y., N. Wakamiya, et al. (2007). Adaptive and reliable multi-path transmission in wireless sensor networks using forward error correction and feedback. Wireless Communications and Networking Conference.

Comroe, R. and D. J. Costello Jr (1984). "ARQ schemes for data transmission in mobile radio systems." Journal on Selected Areas in Communications 2(4): 472-481.

Deb, B., S. Bhatnagar, et al. (2003). Information assurance in sensor networks. 2nd ACM international conference on Wireless sensor networks and applications.

Deb, B., S. Bhatnagar, et al. (2003). ReInForM: Reliable information forwarding using multiple paths in sensor networks. 28th Annual IEEE International Conference on Local Computer Networks.

Eriksson, O., E. Bjornemo, et al. (2011). On hybrid ARQ adaptive forward error correction in wireless sensor networks. 37th Annual Conference on Industrial Electronics Society.

Hurni, P. and T. Braun (2011). "Link-quality aware run-time adaptive forward error correction strategies in wireless sensor networks." IAM, University of Bern, IAM-11-003, Tech. Rep.

Karl, H., M. Löbbers, et al. (2003). A data aggregation framework for wireless sensor networks. Dutch Technology Foundation ProRISC Workshop on Circuits, Systems and Signal Processing, Citeseer.

Kleinschmidt, J. H., W. C. Borelli, et al. (2009). "An energy efficiency model for adaptive and custom error control schemes in Bluetooth sensor networks." AEU-International Journal of Electronics and Communications 63(3): 188-199.

Kleinschmidt, J. H. and W. da Cunha Borelli (2009). Adaptive error control using ARQ and $\mathrm{BCH}$ codes in sensor networks using coverage area information. 20th
International Symposium on Personal, Indoor and Mobile Radio Communications.

Kopke, A., H. Karl, et al. (2005). Using energy where it counts: Protecting important messages in the link layer. 2nd European Workshop on Wireless Sensor Networks.

Liankuan, Z., X. Deqin, et al. (2010). Adaptive error control in wireless sensor networks. IET International Conference on Wireless Sensor Network

Rice, M. and S. B. Wicker (1994). "Adaptive error control for slowly varying channels." IEEE Transactions on Communications 42(234): 917-926.

Taghikhaki, Z., N. Meratnia, et al. (2012). An Error Control Scheme for Delay Constrained Data Communication in a Chain-Based Wireless Sensor Network. The Seventh IEEE International Conference on Broadband and Wireless Computing, Communication and Applications (BWCCA).

Taghikhaki, Z., N. Meratnia, et al. (2013). "On QoS guarantees of error control schemes for data dissemination in a chain-based wireless sensor networks." Sensors \& Transducers Journal 18: 188-202.

Taghikhaki, Z., N. Meratnia, et al. (2013). "A trustbased probabilistic coverage algorithm for wireless sensor networks." Procedia Computer Science 21: 455-464.

Yan-ming, C., X. Yong-jun, et al. (2009). An adaptive fault-tolerant scheme for wireless sensor networks. International Conference on Communications and Mobile Computing.

Yu, K., F. Barac, et al. (2012). Adaptive forward error correction for best effort Wireless Sensor Networks. International Conference on Communications 